\title{
Infrared Spectra of High Polymers. I. Experimental Methods and General Theory
}

\author{
C. Y. Liang, S. Krimm, and G. B. B. M. SutherLand \\ Harrison M. Randall Laboratory of Physics, University of Michigan, Ann Arbor, Michigan
}

(Received October 27, 1955)

\begin{abstract}
The problems involved in obtaining and interpreting the infrared spectra of high polymers are discussed. Experimental methods are described for recording the infrared spectrum, with particular emphasis on the techniques used in the region between 30 and $140 \mu$. The method for obtaining selection rules for high polymer spectra is described and the use of selection rules and other techniques for assigning fundamental frequencies is evaluated. The calculation of the skeletal frequencies of a polymer molecule is discussed in detail.
\end{abstract}

\section{INTRODUCTION}

A LTHOUGH a number of studies have been made of the infrared spectra of various polymer molecules, and some success has been attained in correlating these spectra with the molecular structure of the individual polymer molecules and their arrangement in the bulk material, this branch of infrared spectroscopy is still in a relatively undeveloped state. The reasons are not hard to seek. In the first place, the theoretical interpretation of the spectrum of a large unsymmetrical molecule in the amorphous solid state is a very different proposition from the interpretation of the infrared spectrum of a small symmetrical molecule in the gaseous state, for which strict selection rules are obeyed and where the rotational fine structure can be a very powerful aid in making unequivocal assignments of bands to particular fundamental modes of vibration. In the second place, Raman spectra can usually be obtained readily for small molecules (either in the gaseous or liquid phase) whereas Raman data on polymer molecules are almost nonexistent because of technical experimental difficulties. Consequently, almost no information has been available on the vibration frequencies of polymers below $700 \mathrm{~cm}^{-1}$. Thirdly, the calculation of the normal vibrations of a large polymer molecule with an unsymmetrical repeating unit is a task from which even the most industrious of us shrinks, especially in view of the uncertainty of the magnitude of many of the potential constants required in making such a computation. Yet the importance of this branch of infrared spectroscopy is only too evident. Infrared analysis has been such a powerful tool in exploring the structure of small molecules that there can be no doubt about the desirability of extending the method to large molecules.

We have, therefore, undertaken a systematic study of the infrared spectra of about 25 polymers in an attempt to provide a better foundation for the interpretation of the spectra of such molecules. First, we investigated their spectra in the region between 700 and $70 \mathrm{~cm}^{-1}$ since this would complete the most serious gap in our knowledge. Next, we reinvestigated their spectra in the region between 3500 and $700 \mathrm{~cm}^{-1}$ since discrepancies had been found between the results of earlier workers in this region. As far as possible, oriented samples were used and the infrared dichroism determined between 3500 and $300 \mathrm{~cm}^{-1}$. In a few cases, specimens of a single polymer having varying degrees of crystallinity were studied, since marked differences have been found between the spectrum of the polymer in the amorphous state and that in the crystalline state. Also in a few cases observations were made on deuterated polymers. The polymers were chosen (as far as was practicable) to fall into series closely related in molecular structure since it was hoped that the general correlation between infrared spectrum and molecular structure would facilitate the assignment of fundamental frequencies. Finally, the theory of polymer spectra has been reviewed and an attempt has been made to lay down certain principles which can be used for all polymer spectra.

In this paper we shall discuss (a) the experimental methods used in these investigations, paying special attention to the region between 30 and $140 \mu$, and (b) the basis for the theoretical methods used in interpreting the spectra of polymers in general. In this way we hope to avoid much needless repetition in the succeeding papers dealing with the spectra of individual polymers in considerable detail.

\section{EXPERIMENTAL}

For the region between $2 \mu$ and $4 \mu$ we used a doublebeam spectrometer with a $\mathrm{LiF}$ prism. This instrument is similar in performance to the Perkin-Elmer or Baird double-beam spectrometers but was built in our own workshops under the direction of Professor E. F. Barker and Mr. E. A. Boettner. Between $4 \mu$ and $15 \mu$, we used a Perkin-Elmer Model 21 Spectrophotometer equipped with a $\mathrm{NaCl}$ prism. The spectra between $15 \mu$ and $140 \mu$ were first obtained on a vacuum grating spectrometer. ${ }^{1}$ Later, the region between $15 \mu$ and $30 \mu$ was also scanned on a Perkin-Elmer Model 112 Spectrometer with a $\mathrm{CsBr}$ prism, mainly in order to obtain polarization data in this region. Polarization studies between $2 \mu$ and $30 \mu$ were made using $\mathrm{AgCl}$ and $\mathrm{Se}$ polarizers. Some qualitative measurements on dichroism have been made with the far-infrared spectrometer, using the polarization properties of the gratings. We

\footnotetext{
I S. Krimm and G. B. B. M. Sutherland, Symposium on Molecular Structure and Spectroscopy, Columbus, Ohio, June, 1952
} 
shall now describe the techniques used in the farinfrared in greater detail since this region presents special difficulties.

The far-infrared vacuum grating spectrometer ${ }^{2,3}$ used in this work was originally designed for the investigation of the spectra of vapors under high resolution, and had to be modified in some respects to make it more suitable for this work. The rate of scanning was increased and photographic recording was replaced by pen recording on a Leeds and Northrup Speedomax recorder. The source used was a strip of 0.0008 in. thick chromel metal, $2.75 \times 0.19$ in., heated electrically to about $1000^{\circ} \mathrm{C}$. This was found to be preferable to chromel coated with thorium oxide. Although the coated chromel is a better emitter than uncoated chromel, the increased emission at shorter wavelengths makes the filtering requirements much more stringent. Indeed, the elimination of the unwanted higher orders of the short wavelength radiation is one of the main problems in farinfrared spectrometry.

By using the following technique we were able to reduce the unwanted radiation to less than $1 \%$ at wavelengths below $65 \mu$ and to about $5 \%$ at longer wavelengths. The radiation beam is chopped by a shutter made of one of the alkali halide crystals, and the amplifier of the detector system is tuned sharply to the chopping frequency. The transmission of the alkali halide which is chosen for a particular wavelength region is such that the halide is opaque to the first-order radiation but transparent to the shorter wavelength radiation. Thus, as the halide periodically interrupts the beam, only the first-order long wavelength radiation is detected by the tuned amplifier. By masking part of the open portion of the shutter, a minor chopping effect on the shorter wavelengths due to reflection from the halide surface is eliminated. Further purification is achieved by using the reststrahlen properties of certain crystals. By reflecting the radiation from a suitable crystal placed in the optical path, the ratio of the energy in the first order to that in the higher orders is increased. The final purification is done by using a filter, a material which is more transparent at the long wavelengths than

TABLE I. Spectrometer components for various regions in far infrared.

\begin{tabular}{|c|c|c|c|c|}
\hline $\begin{array}{l}\text { Region } \\
\text { in } \mu\end{array}$ & $\begin{array}{c}\text { Grating } \\
\text { lines/inch }\end{array}$ & $\begin{array}{l}\text { Shutter } \\
\text { material }\end{array}$ & $\begin{array}{l}\text { Reststrahlen } \\
\text { material }\end{array}$ & $\begin{array}{l}\text { Filter in addition } \\
\text { to paraffin }\end{array}$ \\
\hline $15-25$ & 900 & $\mathrm{CaF}_{2}$ & $\mathrm{LiF}$ & none \\
\hline $20-33$ & 900 & $\mathrm{NaCl}$ & $\mathrm{CaF}_{2}$ & none \\
\hline $30-45$ & 600 & $\mathrm{KCl}$ & $\mathrm{NaF}$ & none \\
\hline $40-65$ & 360 & $\mathrm{KBr}$ & $\mathrm{NaCl}$ & none \\
\hline $63-92$ & 133 & $\mathrm{KI}$ & $\mathrm{KBr}$ & $0.5 \mathrm{~mm}$ quartz \\
\hline $90-110$ & 133 & $\mathrm{CsBr}$ (or $\mathrm{KI}$ ) & $\mathrm{KI}$ & $\begin{array}{l}0.5 \mathrm{~mm} \text { quartz } \\
+1 \mathrm{~mm} \text { paraffin }\end{array}$ \\
\hline $100-140$ & 133 & $\mathrm{CsBr}$ (or $\mathrm{KI})$ & TlBr & $\begin{array}{l}0.5 \mathrm{~mm} \text { quartz } \\
+1 \mathrm{~mm} \text { paraffin }\end{array}$ \\
\hline
\end{tabular}

${ }^{2}$ H. M. Randall, Rev. Sci. Instr. 3, 196 (1932).

${ }^{3}$ H. M. Randall, Revs. Modern Phys. 10, 72 (1938). at the short. Paraffin wax has such properties, and a 2-mm thick wax window on the thermopile acts as such a filter. Crystal quartz coated with soot is particularly good at the wavelengths beyond $60 \mu$. The optimum combination of these components depends of course upon the wavelength region to be studied. In Table I are listed the combinations which we have found most satisfactory.

The wavelength calibration for the far-infrared spectrometer was obtained from the water vapor spectrum, ${ }^{4}$ which could be obtained readily by only partially evacuating the spectrometer case. By reducing the pressure within the case to about $0.1 \mathrm{~mm}$ of mercury, the water vapor spectrum was eliminated, and the polymer spectra were obtained under these conditions. All of the spectra were obtained by recording in succession the energy, through a given wavelength region, with and without a sample in the beam. By maintaining a regulated current through the source and using storage batteries for the lamp in the optical amplifier, it was possible to reproduce the conditions under which the background and sample spectra were run. In this way, reliable percentage transmission curves could be obtained. The preparation of the polymer samples will be described in the subsequent papers since this varied somewhat from polymer to polymer.

\section{SELECTION RULES FOR POLYMER SPECTRA}

In order to derive strict selection rules for the vibration spectra of polymers, it is necessary to make two assumptions: (1) that the polymer molecule is infinitely long, and (2) that these polymer molecules have a regular arrangement in a crystalline lattice. Neither of these assumptions is completely correct but it is impossible to make progress without them and any errors due to them can easily be recognized. By investigating polymers of varying molecular weight, it can readily be verified whether the polymer spectrum is a function of chain length. It is our experience that as soon as the polymer contains at least ten repeating units any differences in the spectra of longer polymers are due to frequencies of the end groups (which gradually disappear as the chain length increases) or to branching, i.e., defects in the polymer structure. Similarly, although it is in general very unusual to be able to obtain a high polymer in the form of a single crystal of suitable size for spectroscopic work, it is generally possible to vary the degree of crystallinity in a polymer sample. Thus the spectrum of the wholly crystalline polymer can be inferred with a fair degree of certainty. The differences to be expected between polymers in the crystalline and amorphous states will also be discussed, although here we are on less certain ground.

Consider a crystalline polymer in which the chemically repeating unit of the polymer contains $p$ atoms.

\footnotetext{
${ }^{4}$ Randall, Dennison, Ginsburg, and Weber, Phys. Rev. 52, 160
} (1937). 
Let the unit cell of the crystal contain $r$ separate polymer molecules each of $q$ chemical repeat units. The unit cell then contains pqr atoms. According to the general theory of crystal spectra, the only fundamental frequencies which are potentially active in absorption or scattering are those in which corresponding atoms in the various unit cells move in phase. Thus the maximum number of observable fundamentals will be $3 p q r-3$, ignoring the 3 translational degrees of freedom which give rise to null vibrations of zero frequency. The selection rules for these $3 p q r-3$ fundamentals can be derived from an analysis of the factor group (unit cell group) of the space group of the crystalline polymer by the usual methods of group theory. ${ }^{5}$ In general this will lead to a rather large number of fundamentals allowed in absorption, and correlation with the observed infrared spectrum may not be very obvious. It is usually easier to make temporarily one further assumption, viz., that the interaction forces between polymer chains are so small that the magnitudes of the fundamentals are not greatly changed by this interaction. This is a reasonable assumption since intermolecular forces are well known to be at least an order of magnitude smaller than the intramolecular forces which determine the values of the fundamentals. This allows one to consider as a first step the simpler problem of the spectrum of a single polymer molecule, which may be regarded as a one-dimensional crystal. The maximum number of observable fundamentals now becomes $3 p q-4$, since in addition to the three translational degrees of freedom, we now also ignore rotation of the polymer molecule about its own axis. The selection rules for this simplified model may be derived from an analysis of the factor group of the one-dimensional space group, ${ }^{6}$ and it is legitimate to start the correlation of theory and experiment at this point.

The differences between the spectrum predicted from consideration of the single polymer molecule and that predicted from the true unit cell will be of two kinds. First, certain of the fundamentals of the single polymer molecule will be split into several components in the unit cell treatment. Whether this splitting will be observed in the spectrum depends, of course, upon the magnitude of the interaction forces between molecules in the unit cell, and therefore cannot be predicted without a more detailed normal coordinate analysis. Second, certain modes which are totally inactive for the single polymer molecule may become active in the unit cell treatment. This will happen when the site symmetry of the molecule in the unit cell is lower than the symmetry of the isolated molecule. Since the site symmetry of a molecule in a crystal is usually equal to or lower than the symmetry of the isolated molecule, the only change which can result on considering the complete

\footnotetext{
${ }^{5}$ See, for example, S. Bhagavantam and T. Venkatarayudu, "Theory of Groups and its Application to Physical Problems." Andhra University, Waltair, India. (1951).

${ }_{6}$ M. C. Tobin, J. Chem. Phys. 23, 891 (1955).
}

crystalline arrangement is a relaxation of the selection rules predicted on the basis of the isolated molecule. Therefore, it will never happen that a mode predicted to be active in an isolated molecule is forbidden in the crystal. The reverse situation is, however, possible and must be considered whenever an analysis is made only on the isolated molecule.

It may be useful to point out the advantage which the group theory approach has over the qualitative method of group frequencies previously used in the interpretation of polymer spectra. In the method of group frequencies one associates a band in the spectrum with a separable vibration of a specific chemical group. Although useful in characterizing chemical groups and in identifying polymers, this approach cannot give information on the structural configuration of the polymer. The reason is that when the repeat unit of the polymer contains more than one chemical unit, the normal modes of the polymer are built up essentially by combining group modes according to the various possible phase differences between motions in neighboring groups. The frequency of the resulting fundamental depends upon this phase difference, and so there will be in general more frequencies than would be predicted on the group frequency approach. It is, therefore, only by the detailed analysis of all of the frequencies, which can be accomplished with the aid of the selection rules derived from group theory, that we can expect to obtain structural information about the molecule.

For the polymer molecules in the amorphous state no selection rules are possible. Thus one might expect the spectrum to be much richer than that of the same polymer in the crystalline state. In general this is not true. The spectrum of the amorphous form is generally much more diffuse than that of the crystalline form but bears a rather close resemblance to it. When new absorption bands are found in the amorphous form these may be due either to breakdown of selection rules operative in the crystal or to the existence of different rotational isomers which are absent from the crystalline phase. The diffuseness associated with the spectrum in the amorphous state is probably due to the random nature of the interactions which can occur between individual polymer molecules. In the crystalline state the interactions are precise and the same for each unit cell so that the splitting of a fundamental frequency of the repeat unit of a single polymer is correspondingly well defined. In the amorphous state the great variety of possible intermolecular configurations will lead to an ill-defined broadening of the absorption frequency. Rotational isomerism, which may make it impossible to define a repeat unit for the individual polymer in this phase, can also cause a similar effect since the number of allowed frequencies may now be as high as $3 N$ where $N$ is the total number of atoms in the polymer. However, the majority of these frequencies must be very close to the $3 p q-4$ frequencies of the repeating unit. 


\section{ASSIGNMENT OF FUNDAMENTAL FREQUENCIES}

The selection rules derived from the character tables of the factor group of the space group associated with any crystalline polymer will predict the dichroic properties of the frequencies active in absorption. By itself this does not enable us to make a complete analysis of the spectrum, and a variety of supplementary methods must be used in order to make assignments which have a physical interpretation and are not merely a grouping of observed bands into a few symmetry classes. The following are the most useful guides.

(a) Approximate Normal Coordinate Analysis.-Since the structures of most polymer molecules are either complicated or not known, the normal coordinate analysis cannot in general be applied. In some cases, however, an approximate treatment may be carried out and partial assignment of the observed spectrum of the polymer can thus be made. We have used the following methods of approximation: (1) The normal modes of a single characteristic group in the polymer molecule can be determined by analogy from the analysis of a similar small molecule. Thus, for example, three of the normal modes of a $\mathrm{CH}_{2}$ group in a polymer would be expected to approximate quite closely to those of a similar nonlinear triatomic molecule. (2) If the actual polymer structure can be approximated by a simpler one whose normal frequencies can easily be calculated, then the frequencies of the assumed simple model will be suggestive in the interpretation of the observed spectrum of the actual polymer. This is, for example, true in the case of polytetrafluoroethylene. ${ }^{7}$ (3) By considering only the extended skeleton of the polymer, i.e., taking the component groups as point masses, it is possible to calculate the skeletal frequencies of either a finite or an infinite chain. We will discuss this method in greater detail in the next section.

(b) Group Frequencies,-From the complete analysis of the spectra of small molecules many group frequencies are now well established. This is especially true of hydrogenic stretching frequencies of all types and of $\mathrm{C}-\mathrm{H}$ hydrogenic deformation frequencies. There is still some doubt and controversy about certain $\mathrm{N}-\mathrm{H}$ and $\mathrm{O}-\mathrm{H}$ deformation frequencies. The stretching frequencies of C-halogen bonds are reasonably well established but the situation is not so satisfactory with regard to $\mathrm{C}$-halogen deformation frequencies. The stretching frequencies associated with double or triple bonds between $\mathrm{C}, \mathrm{N}$, and $\mathrm{O}$ are well established. As usual, caution is necessary in assigning bands to group frequencies: such assignments can only be made when it is certain that the frequency is a separable one.

(c) Isotope Effect.-The substitution of isotopes (especially $\mathrm{D}$ for $\mathrm{H}$ ) is very helpful in identifying pure hydrogenic frequencies and the existence of interaction between hydrogenic and skeletal frequencies.

(d) Hydrogen Bonding.-In cases where certain

${ }^{7}$ C. Y. Liang and S. Krimm, Phys. Rev. 98, 1548 (1955). groups are known to be involved in hydrogen bonding, the resulting shifts in frequency due to hydrogen bonding can be used to confirm assignments of frequencies to these groups.

(e) Chemical Substitution.-The replacement of one atom or one group in a polymer by a different group can be very helpful in identifying group frequencies.

When the above methods are combined with selection rules and measurements on dichroism, it is usually possible to make a fairly satisfactory assignment of the frequencies of the polymer molecule. Although the prediction and interpretation of the group frequencies are often straightforward, the determination of the skeletal frequencies is usually more complicated. In order to tackle this very difficult part of the assignment problem, it is necessary to adopt a different approach and to attempt a calculation of the active frequencies of an infinitely long chain polymer in which the size of the repeat unit is variable. This method is outlined in the next section.

\section{THE SKELETAL FREQUENCIES OF A POLYMER MOLECULE}

There have been several attempts to calculate the skeletal frequencies of long chain molecules such as $n$-paraffins. We have found the method suggested by Kirkwood, ${ }^{8}$ and later modified and extended by Pitzer, ${ }^{9}$ is particularly appropriate as a starting point for the discussion of the skeletal frequencies of long chain polymers. ${ }^{10}$ The basic idea of this method is to consider that a planar zig-zag chain of carbon atoms resembles a one-dimensional crystal, so that its vibrations may be investigated by the methods originally developed by Born and Karman ${ }^{11}$ for a one-dimensional chain. Since we have found it desirable to modify Pitzer's treatment in one respect, we shall start by considering a finite linear chain of $N$ atoms. All the modes of vibration (including null vibrations) are given by assuming that the phase difference $(\phi)$ between successive atoms satisfies the equation

$$
\phi=\frac{\pi l}{N-1} \text { where } l=0,1,2, \cdots N-1
$$

The assumption expressed in Eq. (1) is that an integral number of half-wavelength must be completed within the molecule. This is the same physical assumption which Pitzer made but his mathematical formulation of it, viz.

$$
\phi=\frac{\pi l}{N} \text { with } l=1,2, \cdots N-1,
$$

${ }^{8}$ J. G. Kirkwood, J. Chem. Phys. 7, 506 (1939).

K. S. Pitzer, J. Chem. Phys 8, 711 (1940).

${ }^{10}$ Liang, Sutherland, and Krimm, J. Chem. Phys. 22, 1468 (1954).

${ }^{11}$ M. Born and T. V. Karman, Physik. Z. 13, 297 (1912). 
appears to be in error. For instance, the highest value of $l$ does not correspond to a phase difference of $\pi$ between the end atoms of the chain, but rather between points in space beyond the ends of the finite chain. We feel that the condition given by Eq. (1) corresponds more closely to the physical normal mode than that given by Eq. (2), and that the calculated frequencies will therefore be closer to the true values. Moreover, although the omission of $l=0$ is unimportant in a linear molecule (where it corresponds to a translational motion or null vibration) it cannot be ignored in a zigzag chain, as we shall see when we come to consider that case.

Kirkwood and Pitzer were concerned with finding a suitable approximation to all the modes of vibration of a short chain which could justify the use of equations true for an infinitely long chain. Our problem is somewhat different, since we are not concerned with "polymers" such as butane and hexane but with polymers such as polyethylene which can truly be regarded as infinitely long chains. Furthermore, we are concerned with the spectrum of the polymer in the crystalline state. This consideration imposes very stringent conditions on the number of frequencies which can be active in absorption or scattering. Since our object here is to interpret observed frequencies, it should be clear that we will only be concerned with those frequencies in which the unit cells are vibrating in phase. If the crystallographic repeat unit of the polymer chain contains $q$ chemical units, then these frequencies must satisfy the condition (3),

$$
\phi=\frac{2 \pi r}{q} \text { where } r=0,1, \cdots q-1,
$$

in addition to maintaining a constant phase difference between successive chemical units in the chain.

Let us consider a planar zig-zag chain of carbon atoms such as forms the skeleton of many polymers. The onedimensional crystallographic unit cell contains two chemical units $(q=2)$ so that the only potentially active modes are those for which $\phi=0$ or $\pi$. It will be noticed that this agrees with our earlier result that the number of potentially active frequencies in a single polymer chain having $p$ atoms per chemical repeat unit is $3 p q-4$. The vibration pattern for this case is shown in Fig. 1, where rotations and translations have been included to make the general theory clearer. Thus, the phase differences 0 and $\pi$ may be applied to motions in each of the three spatial directions and four of these correspond to null vibrations. It should be added, that in order to determine the phase differences of successive atoms in the chain, one first performs the symmetry operation which moves atom $n$ to position $n+1$ or $n-1$ (in this case, a rotation followed by a translation) and then examines the phase difference between the motion of atom $n$ in its new position and the motion of the atom normally occupying that position. This means that

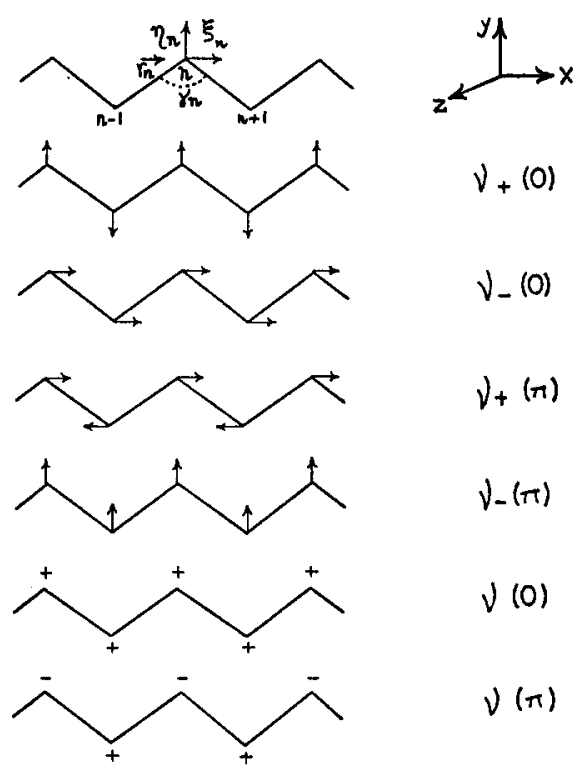

FIG. 1. The skeletal modes of an infinitely long planar zig-zag chain containing 2 carbon atoms in the unit cell.

$\phi=0$ no longer corresponds to translational motions only and so cannot be neglected.

The secular equation of Kirkwood may be applied since we are dealing with an infinite chain. This equation may be written

where

$$
\left|\begin{array}{cc}
K_{11}-\omega^{2} & i K_{12} \\
-i K_{12} & K_{22}-\omega^{2}
\end{array}\right|=0
$$

$$
\begin{aligned}
& K_{11}= \frac{2 K_{c}}{m_{c}} \sin ^{2} \frac{\gamma}{2}(1-\cos \phi)+\frac{2 K_{\alpha}}{m_{c}} \cos ^{2} \frac{\gamma}{2}(1-\cos 2 \phi) \\
& K_{22}=\frac{2 K_{c}}{m_{c}} \cos ^{2} \frac{\gamma}{2}(1+\cos \phi) \\
&+\frac{2 K_{\alpha}}{m_{c}} \sin ^{2} \frac{\gamma}{2}(3+4 \cos \phi+\cos 2 \phi)
\end{aligned}
$$

$K_{12}=2 \sin \frac{\gamma}{2} \cos \frac{\gamma}{2}\left[\left(\frac{2 K_{\alpha}}{m_{c}}-\frac{K_{c}}{m_{c}}\right) \sin \phi+\frac{K_{\alpha}}{m_{c}} \sin 2 \phi\right]$

and

$K_{c}=$ stretching force constant of the $\mathrm{C}-\mathrm{C}$ bond,

$K_{\alpha}=$ deformation force constant of the $\mathrm{C}-\mathrm{C}-\mathrm{C}$ angle,

$m_{c}=$ mass of carbon atom (or mass of $\mathrm{CH}_{2}$ group when calculating the skeletal frequencies of polyethylene),

$\gamma=\mathrm{C}-\mathrm{C}-\mathrm{C}$ angle, assumed to be the tetrahedral angle.

The solution may be written in the form

$$
\omega_{ \pm}^{2}=\omega_{0}^{2} \pm\left(\omega_{0}^{4}-\omega_{1}^{4}\right)^{\frac{1}{2}}
$$




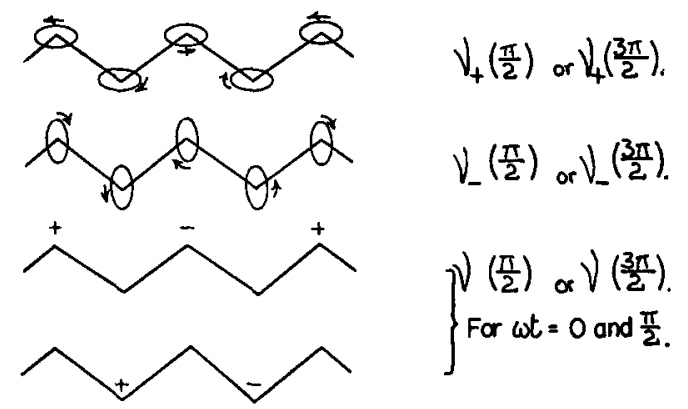

Fig. 2. The skeletal modes in addition to those of Fig. 1 which result when the unit contains 4 carbon atoms.

where

$$
\begin{aligned}
& \omega_{0}^{2}=\frac{K_{c}}{m_{c}}(1+\cos \gamma \cos \phi) \\
& \quad+\frac{2 K_{\alpha}}{m_{c}}(1+\cos \phi)(1-\cos \gamma \cos \phi) \\
& \omega_{1}^{4}=\frac{8 K_{c} K_{\alpha}}{m_{c}{ }^{2}}(1+\cos \phi) \sin ^{2} \phi
\end{aligned}
$$

and

$$
\omega_{ \pm}(\phi)=2 \pi \nu_{ \pm}(\phi) .
$$

In applying these equations to the in plane vibrations of a planar zig-zag chain we have assumed $K_{c}=3.43$ $\times 10^{5}$ dynes $/ \mathrm{cm}$ and $K_{\alpha}=K_{c} / 10=0.34 \times 10^{5}$ dynes $/ \mathrm{cm}$, the value of $K_{c}$ being chosen to make $1 / 2 \pi\left(2 K_{c} / m_{c}\right)^{\frac{1}{2}}$ $=980 \mathrm{~cm}^{-1}$, in approximate agreement with the $\mathrm{C}-\mathrm{C}$ stretching fundamental of ethane. When the unit cell contains 2 carbon atoms (i.e., $q=2$ ) we obtain as the only nonzero frequencies

$$
\nu_{+}(0)=1070 \mathrm{~cm}^{-1} \text { and } \nu_{+}(\pi)=1137 \mathrm{~cm}^{-1} .
$$

The application of this result to the spectrum of polyethylene will be discussed in the succeeding paper.

Next, we consider the case where there are 4 carbon atoms in the unit cell $(q=4)$. There will be twelve modes, six of which are identical with those obtained for $q=2$. The six new modes are illustrated in Fig. 2 . It will be observed that each is doubly degenerate. The degeneracy arises because we have taken a unit of four carbon atoms, which is not one unit cell but a segment consisting of two unit cells. As soon as alternate carbon atoms have a distinguishing characteristic this degeneracy will be removed. The distinguishing characteristic might be the replacement of hydrogen by deuterium in alternate $\mathrm{CH}_{2}$ groups or a similar replacement by a halogen, provided that none of the new frequencies introduced is close to a skeletal $\mathrm{C}-\mathrm{C}-\mathrm{C}$ frequency. In this way we can obtain, at least to a first approximation, the skeletal modes of a large number of the polyvinyl polymers, where the repeat unit contains 4 carbon atoms. This, of course, involves the assumption that the polymer chain is in the fully extended form, which is not necessarily true, but the difference in skeletal frequencies for these two cases is probably small.

We have still to consider the out of plane frequencies. In addition to the null vibrations $\nu(0)$ and $\nu(\pi)$ for the case of $q=2$ we now have the non zero degenerate pair $\nu(\pi / 2)$ and $\nu(3 \pi / 2)$ for $q=4$. These may be calculated using Pitzer's equation

$$
\omega^{2}=4 \pi^{2} \nu^{2}=\frac{K_{3}}{m_{c}}(4-2 \cos \phi-4 \cos 2 \phi+2 \cos 3 \phi) .
$$

Here, $K_{3}$ is the force constant for rotation about the $\mathrm{C}-\mathrm{C}$ bonds and is assumed to be $0.034 \times 10^{5}$ dynes $/ \mathrm{cm}$ to give agreement with the out-of-plane vibration of butane at $140 \mathrm{~cm}^{-1}$, where $\omega^{2}=4 K_{3} / m_{c}$. Using Eqs. (5) and (7) we obtain for $q=4$ the following frequencies as a first approximation to the values of the 5 nonzero fundamentals.

$$
\begin{aligned}
\nu_{+}(0) & =1070 \mathrm{~cm}^{-1} \quad \nu_{+}(\pi)=1137 \mathrm{~cm}^{-1} \\
\nu_{+}(\pi / 2) & =\nu_{+}(3 \pi / 2)=980 \mathrm{~cm}^{-1} \\
\nu_{-}(\pi / 2) & =\nu_{-}(3 \pi / 2)=438 \mathrm{~cm}^{-1} \\
\nu(\pi / 2) & =\nu(3 \pi / 2)=198 \mathrm{~cm}^{-1} .
\end{aligned}
$$

The application of these results to various polymers will be discussed in subsequent papers.

The authors are pleased to acknowledge the benefit of discussing certain points in this paper with Dr. N. Sheppard and Dr. D. M. Simpson. Two of us (S.K. and C.Y.L.) are grateful to the E. I. du Pont de Nemours Company for supporting grants during the tenure of which this work was accomplished.

We are also indebted to M. C. Tobin for the opportunity of reading the manuscript of his paper prior to publication. 